\title{
A Novel Hygrotaxis Assay for Assessing Thirst Perception and Water Sensation in Drosophila Yuanjie Sun ${ }^{1,2, *}$, Shan $\mathrm{Gao}^{1,2}$, Feiteng $\mathrm{Ji}^{1,2, \$}$ and Yan $\mathrm{Zhu}^{1,2}$
}

\author{
${ }^{1}$ State Key Laboratory of Brain and Cognitive Science, Institute of Biophysics, Chinese Academy of \\ Sciences, Beijing, China; ${ }^{2}$ University of Chinese Academy of Sciences, Beijing, China; ${ }^{\$}$ Current address: \\ Peking-Tsinghua Center for Life Sciences, State Key Laboratory of Biomembrane and Membrane \\ Biology, PKU-IDG/McGovern Institute For Brain Research, Beijing Advanced Innovation Center for \\ Genomics, School of Life Sciences, Peking University, Beijing, China \\ *For correspondence: sunyj@ibp.ac.cn
}

\begin{abstract}
[Abstract] Water is essential for the survival of terrestrial animals. Animals obtain water from their food, from metabolism and, more importantly, by drinking. It is therefore vital for dehydrated animals to efficiently locate water sources in the wild. Traditional paradigm for studying the genetic basis of humidity sensation and preference use an apparatus consisting of two chambers providing a binary choice between flows of dry and moist air that maintain steep humidity gradients. Here, we introduce a novel assay to study water-seeking behavior (hygrotaxis) in Drosophila, in which dehydrated flies detect a humidity gradient and rapidly aggregate near an inaccessible water source. Our previous work using this method demonstrated that hygrotactic behavior relies on both specific sensory organs and central brain neurons.
\end{abstract}

Keywords: Hygrotactic behavior, Water-seeking, Water deprivation, Drinking motivation, Drosophila

[Background] Detection of water and regulation of water intake are essential requirements for terrestrial animals to maintain proper osmotic homeostasis (Bourque, 2008; Cameron et al., 2010). Acute loss of one-tenth of body water can result in death in humans, while chronic water imbalance causes multiple disorders and accelerates the aging process (Minassian et al., 1989; Dmitrieva and Burg, 2011 and 2015; Hbibi et al., 2012; Kim et al., 2013). Water-seeking behavior has been well studied in a number of animal groups, but the neural circuits and molecular mechanisms involved in water-seeking are still poorly understood. The experimental method described below could be used to study the genetic mechanisms and neural circuits that regulate hygrotactic behavior, drinking motivation, thirst perception and water sensation in Drosophila.

Preference for specific relative humidities in flies is well established (Perttunen and Erkkila, 1952). Previous studies on humidity sensation in adult flies have generally used a binary-choice chamber ( $T$ maze) (Sayeed and Benzer, 1996; Liu et al., 2007; Montell, 2008). In this design, dry and moist air are circulated to produce and maintain steep humidity gradients (Russell and Pierce-Shimomura, 2014). Recently, Stensmyr and colleagues devised a behavioral assay in which flies were given a choice between different levels of relative humidity (RH) (Enjin et al., 2016). These authors took advantage of the hygroscopic features of super-saturated salt solutions (Winston and Bates, 1960) to create niches with different $\mathrm{RH}$ levels in a multi-well plate. Super-saturated solutions of $\mathrm{LiCl}, \mathrm{NaCl}$, and $\mathrm{KH}_{2} \mathrm{PO}_{4}$ 
Please cite this article as: Sun et. al., (2019). A Novel Hygrotaxis Assay for Assessing Thirst Perception and Water Sensation in Drosophila,Bio-protocol 9 (3): e3154. DOI: 10.21769/BioProtoc.3154.

generated stable humidities of $20 \%, 70 \%$, and $85 \% \mathrm{RH}$, respectively. Using this method, they showed that the preference for a specific humidity range depends on specialized neurons (Enjin et al., 2016). Furthermore, using a similar experimental protocol in which flies were allowed to choose between $20 \%$, $70 \%$, and $85 \% \mathrm{RH}$, Garrity and colleagues identified that IR93a, an ionotropic receptor, is important for hygrosensation in Drosophila (Knecht et al., 2016).

Here, we present a novel assay that we developed to study hygrotactic behaviors in dehydrated flies that have been deprived of water for several hours. A continuous humidity gradient was first established in a chamber with an inaccessible water source. Then, the characteristic behavior of dehydrated flies to rapidly aggregate near a water source was observed. The simplicity of this assay makes it useful for screening a large number of fly strains to investigate hygrotactic behaviors, drinking motivation, thirst perception, and water sensation. This new assay has helped us to identify the synaptic output from the mushroom-body $\alpha / \beta$ surface and posterior neurons as the critical step for both hygrotactic behavior and water sensation (Ji and Zhu, 2015).

\section{Materials and Reagents}

1. $1 \mathrm{ml}$ pipette tips (Haimen Moerde Laboratory Equipment Factory, catalog number: 20180411)

2. Petri dishes, $35 \mathrm{~mm}$ diameter (Corning, catalog number: 430165)

3. Acrylic sheet (Donchamp Science and Technology, thickness: $2.5 \mathrm{~mm}$ )

4. $1.5 \mathrm{ml}$ Eppendorf tubes (Haimen Moerde Laboratory Equipment Factory, catalog number: 20180315)

5. Marker pen (Genvana Industrial Development)

6. Transparent tape (XiaoGan Shushi Group)

7. Nylon mesh (Shanghai Xuhong Screen mesh, model: 100 mesh)

8. Plastic tray $(220 \times 160 \times 60 \mathrm{~mm}, \mathrm{~L} \times \mathrm{W} \times \mathrm{H})$ (Jinwanyi Riyongbaihuo Pifahang)

9. Canton-S fly (Provided by Cold Spring Harbor Laboratory)

10. Cyanoacrylate glue type 502 (Guangdong Aibida Adhesive)

11. Calcium chloride anhydrous $\left(\mathrm{CaCl}_{2}\right)$ (Sinopharm Chemical Reagent, CAS No: 10043-52-4)

12. Ethanol solution (Beijing Chemical Works, catalog number: 112412038320131)

13. Corn meal (Beijing Shouhang Guoli Trading Co., LTD)

14. Yeast (AB MANRI Company)

15. Agar (Beijing Zhongsheng Wanda Biotechnology Co., LTD)

16. Sucrose (Sinopharm Chemical Reagent, catalog number: 10021418)

17. Glucose (Beijing Beihua Fine Chemicals Co., LTD)

18. Potassium sorbate (Ningbo Wanglong Tech Co., LTD)

19. Fly food (see Recipes) 


\section{Equipment}

1. Incubator (Ningbo Jiangnan Instrument Factory, model: HWM-80)

2. Video recorder (Sony, model: HDR-CX405)

3. Stereo microscope (Olympus, model: SZ61)

4. Electric drill (Dremel, model: LITHIUM-10N)

5. $\mathrm{CO}_{2}$ flypad (Beijing Yiran Machine Processing Factory, model: $50 \mathrm{~mm} \times 30 \mathrm{~mm}$ )

6. LED array (Beijing Feiwu Zhenxing Electronic, model: LD-53404CWC03-A)

7. Storage chamber $(265 \times 200 \times 170 \mathrm{~mm}, \mathrm{~L} \times \mathrm{W} \times \mathrm{H})$ (Beijing Xitianlong Plastic Products)

8. Food vials (diameter $32 \mathrm{~mm}$, height $80 \mathrm{~mm}$ ) (Jiangsu Haimen Jayu Experimental Instrument Factory)

9. Culture bottles (diameter $48 \mathrm{~mm}$, height $100 \mathrm{~mm}$ ) (Jiangsu Haimen Jayu Experimental Instrument Factory)

\section{Software}

1. MATLAB (MathWorks, R2013a)

2. Prism 5.0 (GraphPad Software)

\section{Procedure}

A. Construction of chamber and preparation of assay components (Figure 1)

1. Dish for water deprivation

a. Punch a hole in the Petri dish lid

Use the lid of a $35 \mathrm{~mm}$ Petri dish to make the chamber for water deprivation of flies. Punch a $4 \mathrm{~mm}$ diameter hole through the side of each lid with an electric drill.

b. Cover the Petri dish lid with nylon mesh (Figure 1A)

Cut a circle of nylon net (100 mesh) slightly larger than the Petri dish lid. Spread 502 glue evenly round the edge of the lid, and then press the cover onto the nylon net with a heavy weight for at least $15 \mathrm{~min}$.

c. Clean the finished dish

To eliminate glue odors, wash the resulting experimental dish three times with distilled water and dry it in a ventilated space for at least five days before the experiment. 
A

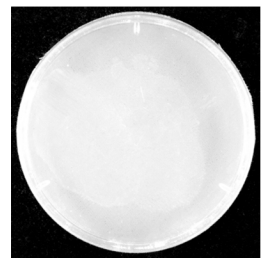

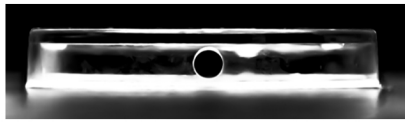

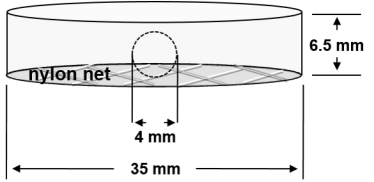

Figure 1. Experimental dish used for water deprivation. A. Photograph of the top view of the dish used for water deprivation. B. Photograph of the side view of the dish used for water deprivation. C. Dimensions of the experimental dish used for water deprivation.

2. Construction of the dry chamber for water deprivation of flies (Figure 2)

a. Make the dry box

Place $600 \mathrm{~g}$ anhydrous $\mathrm{CaCl}_{2}$ into a plastic tray $(220 \times 160 \times 60 \mathrm{~mm}, \mathrm{~L} \times \mathrm{W} \times \mathrm{H})$.

b. Cut the acrylic sheet

Cut a rectangle of acrylic sheet to cover the plastic tray containing $\mathrm{CaCl}_{2}(240 \times 180 \times 3 \mathrm{~mm}$, $\mathrm{L} \times \mathrm{W} \times \mathrm{H})$.

c. Construct the dry chamber

Transfer the covered plastic tray into a storage chamber $(265 \times 200 \times 170 \mathrm{~mm}, \mathrm{~L} \times \mathrm{W} \times \mathrm{H})$.

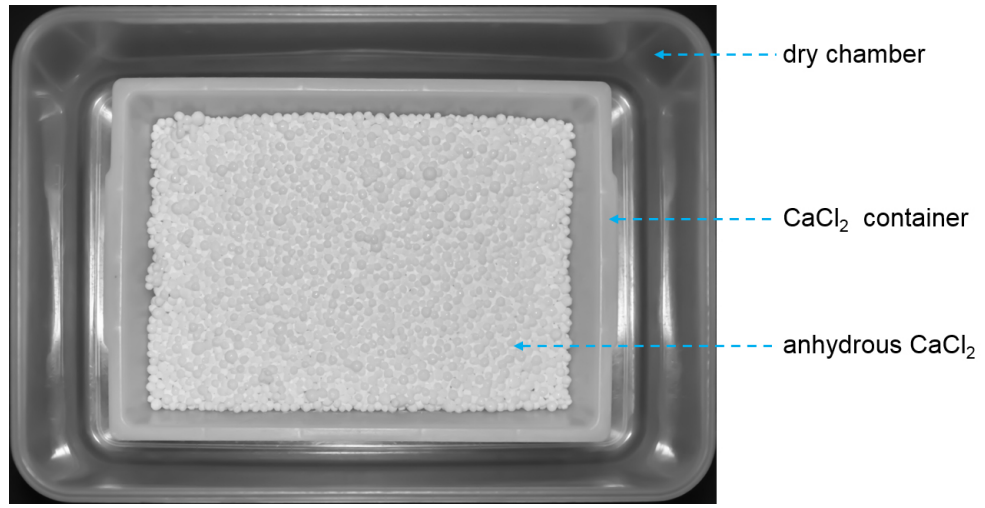

Figure 2. Drying chamber for water deprivation. Anhydrous calcium chloride $(600 \mathrm{~g})$ is contained in a covered plastic tray, which is kept in a storage chamber.

B. Make the humidity plate (Figure 3)

1. Drill holes in an acrylic sheet

Use an acrylic sheet with a thickness of $2.5 \mathrm{~mm}$ to make the humidity plate. Cut the sheet into rectangular pieces $300 \times 200 \mathrm{~mm}$. Drill ten $9 \mathrm{~mm}$ holes through each sheet (ensure the distance between holes is at least $70 \mathrm{~mm}$, as shown in Figure 3B).

2. Attach caps of Eppendorf tubes to surround the holes

Remove the caps from $1.5 \mathrm{ml}$ Eppendorf tubes. Apply 502 glue to these caps and press them onto the acrylic sheet to surround the holes, for $5 \mathrm{~min}$. Leave the sheet overnight before the next step.

3. Mark the positions of the experimental dishes on the humidity plate 
When the Eppendorf tube caps are tightly adhered to the acrylic sheet, draw a circle with a diameter of $40 \mathrm{~mm}$ using a marker pen with each hole as the center.

4. Clean the humidity plate

To eliminate glue odor, wash the humidity plate with distilled water three times and dry in a ventilated place for at least five days before the experiment.
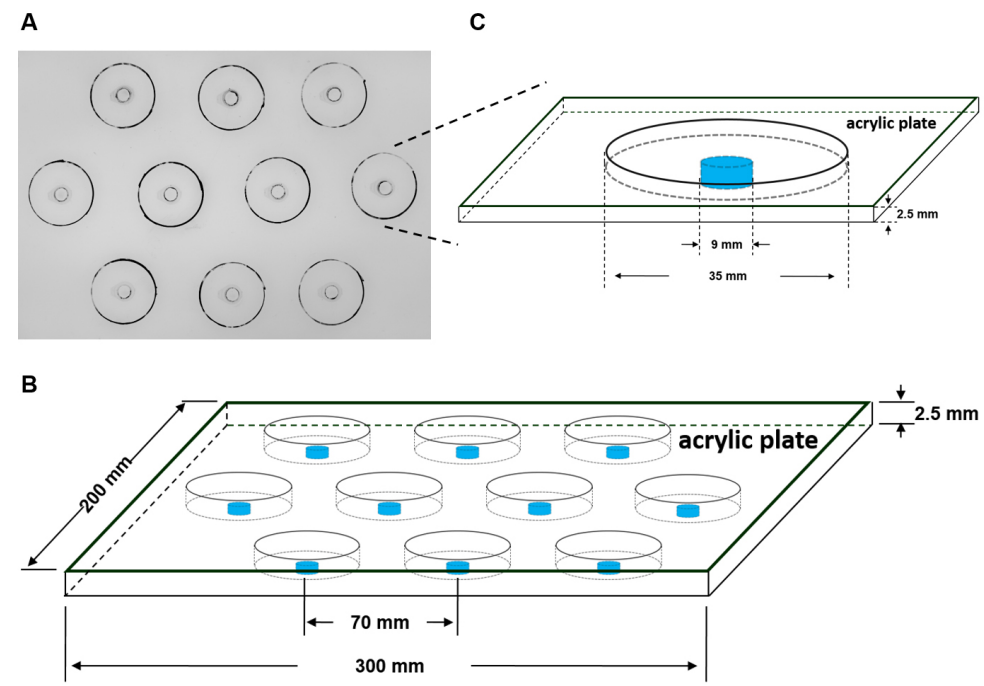

Figure 3. Humidity plate. A. Plan view of a photograph of the humidity plate. B. Diagram of the humidity plate with dimensions. C. Diagram of one hole of the humidity plate with dimensions.

\section{Preparation of flies}

\section{Fly Stocks}

Maintain flies at $25{ }^{\circ} \mathrm{C}$ and $60 \% \mathrm{RH}$ under a $12: 12 \mathrm{~h}$ light/dark cycle. Use adult flies 4-7 days after eclosion for hygrotactic assays.

\section{Fly rearing}

To maintain a suitable population density, transfer 20 females and 10 males of wild-type flies to a culture bottle (diameter $48 \mathrm{~mm}$, height $100 \mathrm{~mm}$ ) and allow females to lay eggs for two days; then discard the adult flies.

3. Preparation of flies for behavioral tests

Collect 20 male (or female) flies on a $\mathrm{CO}_{2}$ fly pad under a stereo microscope into a fresh food vial (diameter $32 \mathrm{~mm}$, height $80 \mathrm{~mm}$ ). Maintain the collected flies at $25^{\circ} \mathrm{C}$ and $60 \% \mathrm{RH}$ in the food vial under a 12:12 h light/dark cycle for two days before tests.

D. Water deprivation (Figure 5)

1. Transfer flies into a water deprivation dish

Gently move the 20 flies from their food vial into the tip of a $1 \mathrm{ml}$ pipette with an attached suction tube (Figure 4), and then gently blow the flies into a water-deprivation dish through its side hole. Seal the hole with transparent tape. 


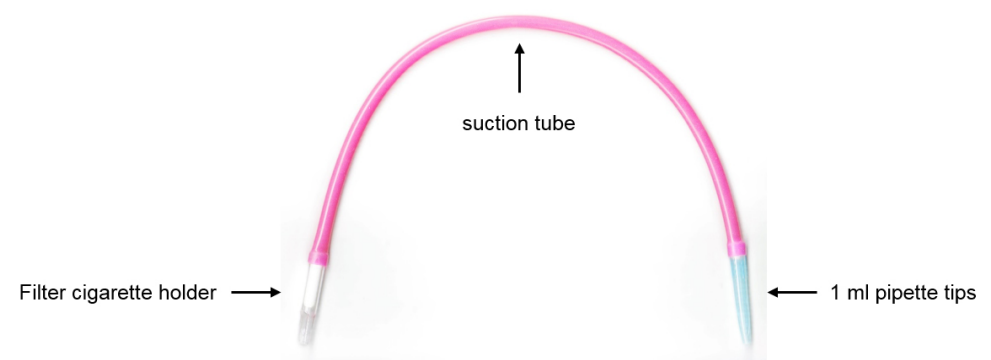

Figure 4. Suction tube. Photograph of the top view of the suction tube for fly transfer from a culture tube into a water-deprivation dish.

2. Transfer the experimental dishes into the dry chamber

Gently position the water-deprivation dishes loaded with flies onto the acrylic sheet in a dry chamber with the nylon net facing upward (Figure 5A).

3. Place the dry chambers in an incubator

Seal the dry chambers by closing their lids. Transfer the dry chambers into an incubator at $25^{\circ} \mathrm{C}$ and $60 \% \mathrm{RH}$ (Figure $5 \mathrm{~B}$ ) for $8 \mathrm{~h}$.

A

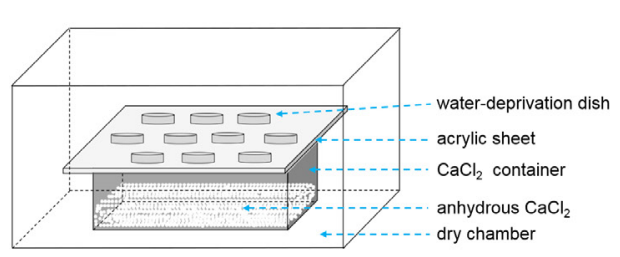

B

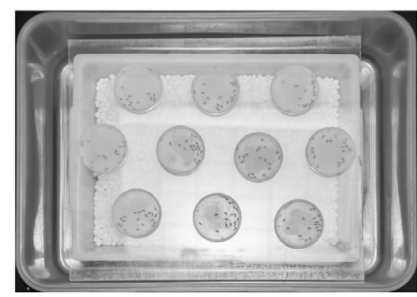

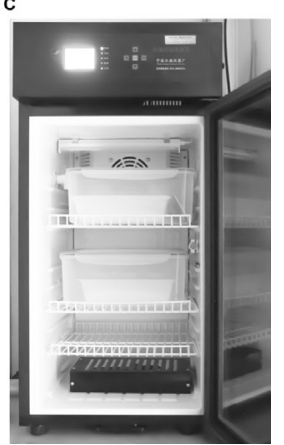

Figure 5. Procedure for water deprivation. A. A schematic side view of water deprivation dishes covered with 100 mesh nylon net in a dry chamber. B. Photograph of a water-deprivation dish loaded with flies in the dry chamber. C. Photograph of dry chambers in an incubator.

E. Observation of hygrotactic behavior in Drosophila

1. Set the testing environment

Before the behavioral experiment, set the room temperature to $24 \pm 1^{\circ} \mathrm{C}$ and the humidity to $40-60 \% \mathrm{RH}$.

2. Add water to the humidity plate

Place the humidity plate on a horizontal surface. Add $200 \mu$ distilled water to fill each cap on the plate and allow the plate to equilibrate at room temperature $\left(24 \pm 1^{\circ} \mathrm{C}\right)$ for $30 \mathrm{~min}$.

3. Environmental acclimatization of flies

After water deprivation, gently remove the experimental dishes with flies from the dry chamber. Allow the flies (still in their dishes) to acclimate to the experimental environment, including temperature $\left(24 \pm 1^{\circ} \mathrm{C}\right)$, humidity $(40-60 \% \mathrm{RH})$, and illuminance (we use an array of white LEDs as the light source, $800-850$ Lux) for $15 \mathrm{~min}$. 
4. Record water-seeking behavior (see Video 1)

Turn on the video recorder and adjust the focus to the top surface of the humidity plate; then start recording. Gently place the experimental dishes on the humidity plate with the nylon net side facing downward. Align the experimental dishes with the centers of the water-containing caps, one by one. A video recorder monitors ten dishes on one humidity plate. Turn off the video recorder after the last dish has been placed on the humidity plate for $5 \mathrm{~min}$.

Note: The control group (flies without water deprivation) and experimental group (flies with water deprivation) should be placed on the same humidity plate.

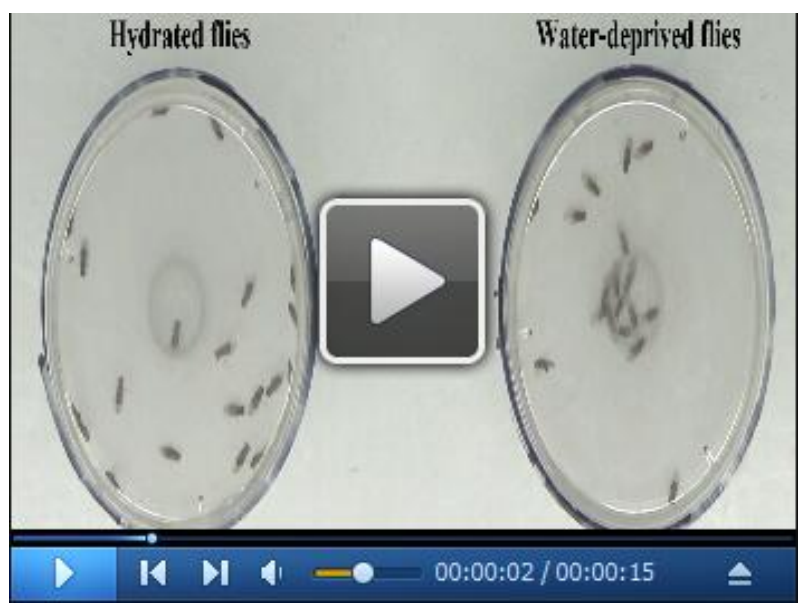

Video 1. Recording of water seeking flies. The left dish contains water-deprived flies. The right dish contains hydrated flies. The video is played at a speed of four times of normal.

F. Cleaning experimental dishes and chambers

The dry chamber should be washed after use with distilled water and/or dilute ethanol solution ( $70 \%)$. The water-deprivation dish should only be washed with distilled water. We strongly recommend wearing gloves when handling deprivation dishes and chambers to prevent transfer of unwanted odors.

\section{Data analysis}

1. To quantify the hygrotactic behavior, we defined a circular region of diameter $17.5 \mathrm{~mm}$ in the center of the dish and counted the number of flies in this region at different time points (Figure 6). The 'hygrotaxis index' was calculated using a script written in MATLAB as follows. Firstly, we calculated the hygrotaxis values at different time points during the test. The hygrotaxis values were calculated using the following formula:

Hygrotaxis Value $\left(H V_{t}\right)=\left(N_{t}-N_{0}\right) / N_{\text {sum }}$ where, 
$\mathrm{Nt}$ : the number of flies in the defined region at this time; No: the number of flies in the region at the beginning of experiment; $\mathrm{N}_{\text {sum: }}$ the total number of flies in the dish. In our tests: $\mathrm{N}_{\text {sum }}=20$. The hygrotaxis index is defined as the average of all aggregation values measured at fivesecond intervals within a 5 min test \{hygrotaxis index $\left.=\left(\mathrm{HV}_{5 \mathrm{~s}}+\mathrm{HV}_{10 \mathrm{~s}}+\mathrm{HV}_{15 \mathrm{~s}}+\ldots+\mathrm{HV}_{300 \mathrm{~s}}\right) / 60\right\}$ Note: For the flies positioned across the inner circle, if the head of fly towards to the inside, the fly was deemed to in the inner circle.
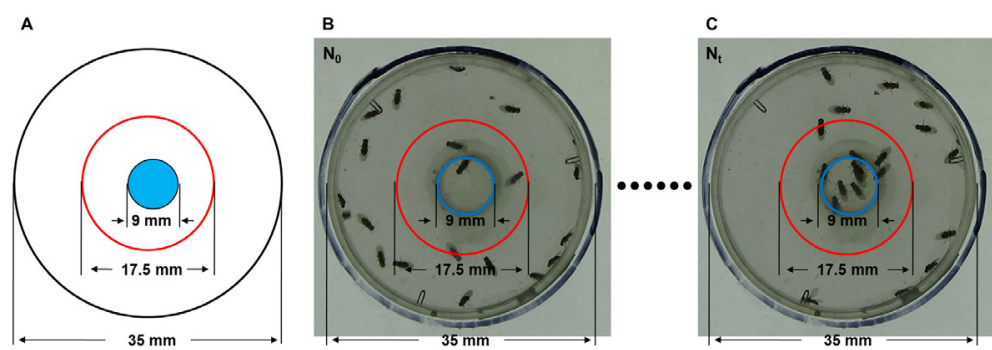

Figure 6. Thirsty flies exhibit hygrotactic behavior. A. Diagram of the zones used to quantify the hygrotactic response for calculation of the hygrotactic index. The dish was placed above a water-containing hole, leaving a gap of $2.5 \mathrm{~mm}$ between the nylon net and the water surface. $\mathrm{B}$. Photograph showing the distribution of flies at time zero. C. Thirty seconds later, the dehydrated flies quickly migrated to and aggregated above the water source.

2. Hygrotaxis index data were entered into Prism for statistical analysis and graphical visualization. The statistical significance of differences in hygrotactic responses between different experimental groups was calculated. Unpaired $t$-tests were used to compare differences in the hygrotaxis indices of hydrated and dehydrated flies (Figure 7).

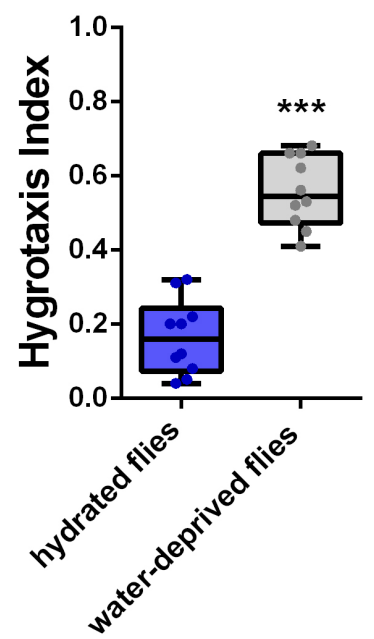

Figure 7. Quantification of hygrotactic behavior in Drosophila. The hygrotaxis index was greatly increased in wild-type flies (Canton-S strain) after water deprivation for $8 \mathrm{~h}$. The middleline in the square represents mean of the distribution, the box represents $25 \%-75 \%$ of the distribution and dots represent average hygrotaxis index of one experiment (more than 5 dishes). 
Please cite this article as: Sun et. al., (2019). A Novel Hygrotaxis Assay for Assessing Thirst Perception and Water Sensation in Drosophila,Bio-protocol 9

\section{Recipes}

1. Fly food (1 L, pH 7.2-7.4)

$77.7 \mathrm{~g}$ corn meal

$32.19 \mathrm{~g}$ yeast

$5 \mathrm{~g}$ agar

$0.726 \mathrm{~g} \mathrm{CaCl}_{2}$

$31.62 \mathrm{~g}$ sucrose

$63.2 \mathrm{~g}$ glucose

$2 \mathrm{~g}$ potassium sorbate

\section{Acknowledgments}

This work was supported by National Natural Science Foundation of China (31771173).

\section{Competing interests}

The authors declare no competing financial interests.

\section{References}

1. Bourque, C. W. (2008). Central mechanisms of osmosensation and systemic osmoregulation. Nat Rev Neurosci 9(7): 519-531.

2. Cameron, P., Hiroi, M., Ngai, J. and Scott, K. (2010). The molecular basis for water taste in Drosophila. Nature 465(7294): 91-95.

3. Dmitrieva, N. I. and Burg, M. B. (2011). Increased insensible water loss contributes to aging related dehydration. PLoS One 6(5): e20691.

4. Dmitrieva, N. I. and Burg, M. B. (2015). Elevated sodium and dehydration stimulate inflammatory signaling in endothelial cells and promote atherosclerosis. PLoS One 10(6): e0128870.

5. Enjin, A., Zaharieva, E. E., Frank, D. D., Mansourian, S., Suh, G. S., Gallio, M. and Stensmyr, M. C. (2016). Humidity sensing in Drosophila. Curr Biol 26(10): 1352-1358.

6. Hbibi, M., Abourazzak, S., Babakhouya, A., Boubou, M., Atmani, S., Tizniti, S. and Bouharrou, A. (2012). Severe hypernatremic dehydration associated with cerebral venous and aortic thrombosis in the neonatal period. BMJ Case Rep 2012: bcr0720114426.

7. Ji, F. and Zhu, Y. (2015). A novel assay reveals hygrotactic behavior in Drosophila. PLoS One 10(3): e0119162.

8. Kim, D., Mun, J. B., Kim, E. Y. and Moon, J. (2013). Paradoxical heart failure precipitated by profound dehydration: intraventricular dynamic obstruction and significant mitral regurgitation in 
Please cite this article as: Sun et. al., (2019). A Novel Hygrotaxis Assay for Assessing Thirst Perception and Water Sensation in Drosophila,Bio-protocol 9

a volume-depleted heart. Yonsei Med J 54(4): 1058-1061.

9. Knecht, Z. A., Silbering, A. F., Ni, L., Klein, M., Budelli, G., Bell, R., Abuin, L., Ferrer, A. J., Samuel, A. D., Benton, R. and Garrity, P. A. (2016). Distinct combinations of variant ionotropic glutamate receptors mediate thermosensation and hygrosensation in Drosophila. Elife 5: e17879.

10. Liu, L., Li, Y., Wang, R., Yin, C., Dong, Q., Hing, H., Kim, C. and Welsh, M. J. (2007). Drosophila hygrosensation requires the TRP channels water witch and nanchung. Nature 450(7167): 294298.

11. Minassian, D. C., Mehra, V. and Verrey, J. D. (1989). Dehydrational crises: a major risk factor in blinding cataract. Br J Ophthalmol 73(2): 100-105.

12. Montell, C. (2008). TRP channels: it's not the heat, it's the humidity. Curr Biol 18(3): R123-126.

13. Perttunen, V. and Erkkila, H. (1952). Humidity reaction in Drosophila melanogaster. Nature 169(4289): 78.

14. Russell, J. and Pierce-Shimomura, J. T. (2014). Apparatus for investigating the reactions of softbodied invertebrates to controlled humidity gradients. J Neurosci Methods 237: 54-59.

15. Sayeed, O. and Benzer, S. (1996). Behavioral genetics of thermosensation and hygrosensation in Drosophila. Proc Natl Acad Sci U S A 93(12): 6079-6084.

16. Winston, P. W. and Bates, D. H. (1960). Saturated solutions for the control of humidity in biological research. Ecology 41(1): 232-237. 